\title{
A Knowledge Representation Model using Concept-Relation Graph
}

\author{
Praveena Rachel Kamala S., Justus S.
}

\begin{abstract}
Huge volume of relevant and irrelevant information is available from different sources for gaining knowledge about a system. If the required data is in a structured form, then the fact can be easily understood otherwise the process becomes laborious and results are vague after intense analysis. In this paper, we are proposing a framework for fetching knowledge from unstructured source of data. The algorithms proposed identifies the concepts and separates the concepts and relation words, enables to add new concepts and also modify the old concept word with new concept and locate the concept. This Concept-Relation Model enables the system to work according to the users' connivance and deliveries accurate knowledge. This model does not manipulate or interpret the information provided but only represent and share the desired knowledge.
\end{abstract}

Index Terms: Knowledge Representation, Logic, Visualization.

\section{INTRODUCTION}

With the advent of big data and IoT data, knowledge engineered intelligent and smart systems are in need for knowledge representation models that would answer the end users' queries. The existing knowledge representation models use one or combination of first-order-logics (FOL), petri-net, concept-maps, frames etc. These models are quite useful when the data size is small and static, but do not address a growing dataset with variety of data flowing in at different velocity [1]. The regular procedures for acquiring knowledge are focused more on capturing atomic concepts and knowledge units (KU) but the redundant $\mathrm{KU}$, and the relationships among the KUs and concepts are insufficient. Redundancy brings to gather similar KU under various categories or domains. Searching and sharing of KU turns out to be more mind boggling and confounded because of these winds up in conveying confused responses for the question posted by the user.

Another open issue is choosing a more suitable storage and retrieval of knowledge units. As far as representation, a precise structure is required for adapting information into a knowledge unit and codifies it into a functional model for intelligent systems. The challenge is how these KUs can be made persistent by storing in a more suitable storage structure and better way of retrieving them so as to answer the end users' query in a more effective way.

The knowledge shared to the user from the acquired information is done by pre-training the system or predicting

\footnotetext{
Revised Manuscript Received on December 02, 2019

Praveena Rachel Kamala S., Research Scholar, School of Computing Science and Engineering, VIT University, Chennai, India.

Justus S., Associate Professor, School of Computing Science and Engineering, VIT University, Chennai, India.
} issues. Nonappearance of relationship between concepts

the outcome through experiences. In that situation if any misassumption or new scenario occurs then the knowledge cannot be generated.

To beat these disadvantages, a point by point study on all the available models and techniques was carried out earlier. As a result of the study, a new knowledge representation model is proposed, called a concept-relation graph. We have focused on an orderly approach to translate the data, and cautiously setting it in an efficient structure withstanding the rules and restrictions.

In this paper, the process involved in representing knowledge is discussed in Section 2. In Section 3, a knowledge representation model using description logic and conceptual graph is illustrated. In Section 4, the four key algorithms for content parsing, adding nodes, modifying nodes and traversing nodes are explained with complexity. In Section 5, conclusion and future work is given for the knowledge representation model designed.

\section{PROCESSES IN REPRESENTING KNOWLEDGE}

Our proposed models for representing knowledge utilize the logical concepts like predicate logics, and first order logic for the information to be deciphered. Description logic is connected to form rules and restrictions. The acquired concepts are mapped to a graphical structure to decide the relationship between every condition. This empowers the development of Concept Relation (CR) diagram to be simple.

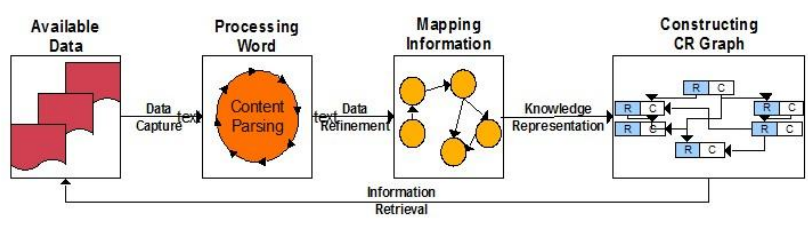

Fig. 1: A Model for Representing Knowledge

Fig. 1 clarifies the general working of the system. Initially the content is received from the available source, in this case, from a structured repository, and the content is processed. The identified concepts are then connected together by means of relations between them. These relations determine the semantics of the two concepts it connect. Our model supports structured and semi-structured form of information for processing and knowledge acquisition. After the information is fed into the structure, content parsing is done where each and every word in processed for identifying it as a concept or a relation that connects two concepts. 


\section{A Knowledge Representation Model using Concept-Relation Graph}

Conflicts in validating the concepts and its relations are carried through logical entailment algorithms. After resolving the conflicts, the graph is constructed using the algorithms we have proposed in this paper.

Conceptual Graph (CG)[2] idea composed by John Sowa[3], defines the conceptual form of knowledge representation. The concepts are represented to as nodes in the diagram having logic based relationship. A CG based knowledge representation encourages a clear and organized approach for codifying the information. CG additionally allows a more precise move towards reasoning. The graphical plan of CG empowers the user to plainly screen the structure and operational technique of the system. Studying the benefits of $\mathrm{CG}$, we have fused this graphical concept into our model for greater clarity, functional structure, logical entailment, and for user assistance.

\section{REPRESENTING USING DL AND CG}

In this Model, the knowledge represented is done by converting the statement into description logic using first order logic[4]. Rules are generated and suitable reasoning is done by applying conceptual graphs structure. Accordingly required output is obtained from the diagram.

Consider this as input statement: "If no man is reading the book, then is there someone who wrote the book reads it?"

\section{Step 1:}

The given statement is converted into logical statements using first order logics (FOL).

1. FOL for the given statement "If no man is reading the book, then is there someone who wrote the book reads it?"

$\forall \mathrm{x}[\operatorname{man}(\mathrm{x}) \wedge \neg \operatorname{Read}(\mathrm{x}$, book $)] \supset \exists \mathrm{x}[$ Author $(\mathrm{x}$, book $) \wedge$ $\operatorname{Read}(\mathrm{x}$, book)]

2. No man reads the book.

$\forall \mathrm{x}[\neg \operatorname{man}(\mathrm{x}) \wedge \operatorname{Read}(\mathrm{x}$, book $)]$

3. Adult is a group containing men and women. (if men are not reading then women are reading)

$$
\forall \mathrm{x}[\operatorname{adult}(\mathrm{x}) \supset \operatorname{man}(\mathrm{x}) \wedge \operatorname{woman}(\mathrm{x})]
$$

4. Woman reads the book.

$\forall x[\operatorname{adult}(x) \supset w o m a n(x) \wedge \operatorname{Read}(x$, book $)]$

5. Assuming negation approach: The author does not read the book.

$\forall \mathrm{x}, \forall \mathrm{y}[$ Author $(\mathrm{x}, \mathrm{y}) \supset \neg \operatorname{Read}(\mathrm{y}, \mathrm{x})]$

6. The author of the book is not Jane.

(y=Jane) $\forall \mathrm{y}[$ woman(y) $\wedge \mathrm{y} \neq$ Jane $\supset$ Author(book,y)]

7. A woman, who is not Jane, does not read the book. (from 5)

$\forall \mathrm{y}[\operatorname{woman}(\mathrm{y}) \wedge \mathrm{y} \neq \operatorname{Jane} \supset \neg \operatorname{Read}(\mathrm{y}$, book $)]$

8. Conclusion: Jane is the author.

Step 2:
General properties for different concepts can be given by using restrictions and distinguish primitive definitions. The Primitive concepts are: Adult $\sqsubseteq \mathrm{T}$, Woman $\sqsubseteq$ Adult, Man ᄃAdult, book $\subseteq\left\{{ }^{\prime}\right\}$

Defined Concepts:

1. [ALL :Man [FILLS :hasn't Read]] ------ > From 2 of Step 1

2. [ALL :Adult[AND Man, Woman]] ------ > From 3 of Step 1

3. [AND Woman [FILLS :has Read]] ------ > From 4 of Step 1

4. [AND Author [FILLS :hasn't Read]] ------ > From 5 of Step 1 (negation approach)

5. [ALL :Woman[EXISTS 1 :Woman (Jane - > [FILLS :can't_be Author])[FILLS :can_be Author]]] ------ > From 6 of Step 1

6. [ALL :Woman[EXISTS 1 :Woman (Jane - > [FILLS :can't_be Author]) [FILLS :hasn't Read]] ------ > From 7 of Step 1

7. [ALL :Woman [EXISTS 1 :Woman [ALL :except Jane] [FILLS :can't_be Author]] ------ > From 5 and 6 of Step 2

8. [AND Woman [FILLS :Jane Author] ------ > From 7 of Step 2 (conclusion).

\section{Step 3:}

Using the theory of conceptual graphs, the information is mapped using the concept - relation graph. Consider the Fig. 2 , which gives the conceptual structure for the above mentioned statement.

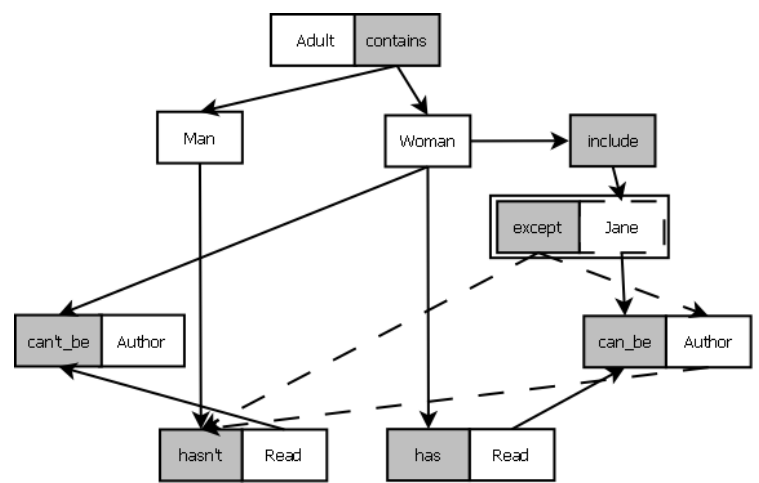

Fig. 2: Concept-Relation Graph for Author - Reader example

In this CR graph, the assumptions are indicated by using a double dash border node as in case of Jane. Since the assumed concept along with the relation signifies a negative approach, they are indicated by a double bordered node.

\section{Step 4:}

The concept along with their relationship is represented as a single node. Mapping between nodes enable the graph to reason the sequence of action.

From the above CR graph, the problem is defined as Adult contains both Men and Women. Since no man reads the book, only a woman can be the Author of the book. By introducing a constant Jane under the category Woman and by applying negation approach to the situation, the conclusion is 
"Jane is the author of the book".

\section{THE ALGORITHMS FOR CONSTRUCTING CONCEPT-RELATION GRAPH}

A few algorithms are designed for working with the graphical structure. The distinctive operations done in a Concept-Relation (CR) diagram are including new nodes, modifying the current nodes and finding the presence of the desirable node. The design used for recovering the knowledge from the graphical structure resembles an attractive magnetic ball impact. At the point when a concept is searched and discovered, at that point all the related concepts (nodes) will be brought relying on their level of relationship. Therefore the retrieval of the stored concept is completed by following the path of their relation. Our Model includes different stages in creating significant extraction of knowledge. This is accomplished by handling the information through various levels. The working methodology and algorithms is discussed below.

\section{A. Algorithm for Content Parsing}

This technique for capturing data [5] assumes a crucial part in knowledge representation [6]. The meaning of the source ought to be same while obtaining the facts. There ought to be no loss of records. The area of storage should be simple bringing of data for further manipulation.

\section{Notation:}

Given a sentence ' $S$ ', containing data ' $D$ ' with concepts ' $C$ ' and relations ' $\mathrm{R}$ '.

Such that $S: D \rightarrow\{C$ U R $\}$, where $C=\left\{C_{1} U_{2} U \cdots . C_{n}\right\}$ and $R=\left\{R_{1} U R_{2} U \cdots . . R_{m}\right\}$

\section{Algorithm:}

Content_Parsing(word_document)

Input: Data: $\left\{\mathrm{D}_{1} \mathrm{UD}_{2} \mathrm{UD}_{3} \mathrm{U} \ldots \mathrm{D}_{\mathrm{k}}\right\}=\left\{\mathrm{C}_{1} \mathrm{UR}_{1} \mathrm{UC}_{2} \mathrm{U} \mathrm{R}_{2} \mathrm{U} \ldots \mathrm{C}_{\mathrm{n}}\right.$ U...... $\mathrm{R}_{\mathrm{m}}$ \}

Given: A set of predefined Relation, Concept Table (CT) and Relation Table (RT)

Output: Parsed content in Sentence Table (ST)

$\left\{\mathrm{C}_{1} \mathrm{UC}_{2} \mathrm{U} \ldots \mathrm{C}_{\mathrm{n}} \mathrm{UR}_{1} \mathrm{U} \mathrm{R} \mathrm{R}_{2} \mathrm{U} \ldots \ldots \mathrm{R}_{\mathrm{m}}\right\}$

\section{Procedure:}

If Data!= NULL

Add Data in Table with s_id

$\mathrm{Ch}=\mathrm{COUNT}($ Data $)$

While $(!=\mathrm{Ch})$

For cmp 0 to $\mathrm{Ch}$

If Dcmp€ Relation

Check DcmpinRT

If Dcmp € RT

Add Dcmpin ST with $r_{-}$id

Else

Add Dcmpin ST with new $r$ id

Else

Check Dcmpin CT

If Dcmp € CT

Add Dcmpin ST with c id

Else

Add Dcmpin ST with new c_id
Else

Repeat till Ch

Print "Enter Valid Value"

In this algorithm Content_Parsing, the word record from where information must be taken is dealt with as the input This record is opened; every word of the lines in the document are perused and stored in the short-term memory. These data are part word by word and recorded in the memory. A spreadsheet is opened [7] and the records are included by composing the split words onto diverse cells in column wise. At that point the recently made spreadsheet is updated for later use. These captured words are included in the sentence table for computational reason.

A unique number is created for the word present and a sequence number is additionally produced as the words must be organized in correct arrange. The information captured is investigated by checking the content report containing the list of relations defined. If the word is already defined in relation list then it is treated as relation otherwise it has to verify with the list of concepts enrolled in the concept table. If the concept is already present then the existing unique number is fetched and used. Adding new concepts to the list requires new unique number.

The Concept Table is created, if no concepts are as of now present. Updating of Concept Table is done only if a single or more concepts are available and just if the concept is absent prior. If the concept previously exists then the unique number is retrieved for completing the sentence table.

The outcome of this algorithm involves creation of sentence table containing the statement from the record given and updating the sentence table with the word, identification of the word as concept or relation, generation of unique number regarding concept and relation and sequence number demonstrating the order of arrangement for simpler computation.

\begin{tabular}{|c|l|}
\hline S_id & \multicolumn{1}{c|}{ Sentence } \\
\hline 175 & It is a very old maxim that "Health is Wealth". \\
\hline 176 & A healthy person can work with efficiency to earn wealth. \\
\hline 177 & $\begin{array}{l}\text { The man who is not having a good health spends lot of money on } \\
\text { medicines and doctors. }\end{array}$ \\
\hline 178 & He is never cheerful. \\
\hline 179 & Which in itself is a wealth? \\
\hline 180 & Butwe have to take precautions for building up a good health. \\
\hline 181 & Healthy body is always dependent on the healthy mind. \\
\hline 182 & $\begin{array}{l}\text { For maintaining goodhealth we should always be away from the } \\
\text { badhabits like smoking, drinking and drug addiction. }\end{array}$ \\
\hline 183 & $\begin{array}{l}\text { We should have morning walk regularly, light exercise in fresh air } \\
\text { and a stroll after dinner. }\end{array}$ \\
\hline 184 & We should not be jealous of others. \\
\hline 185 & We should always take balanced and nutritious diet. \\
\hline 186 & $\begin{array}{l}\text { One should always develop the habits of cleanliness to keep } \\
\text { healthy. }\end{array}$ \\
\hline
\end{tabular}

Fig. 3: Content Stored in Sentence Table with Sentence ID 


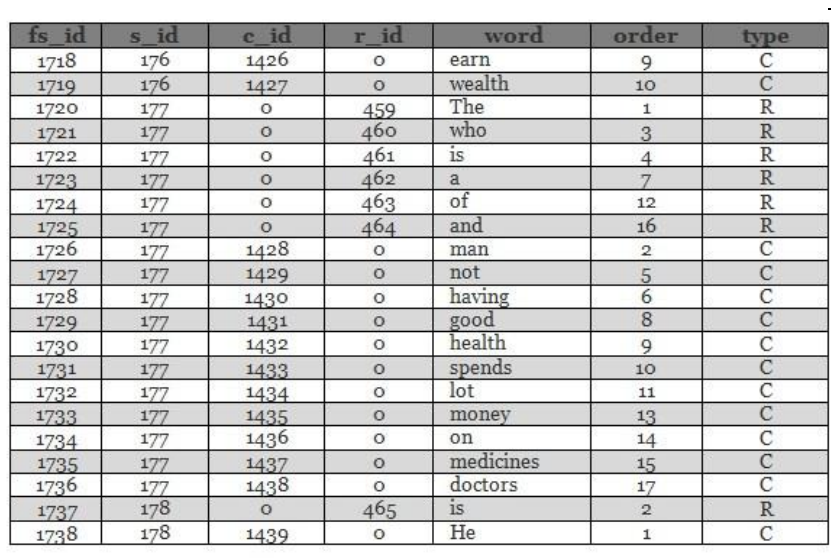

Fig. 4: Parsed Content stored in Table

\section{Complexity:}

Assume that ' $n$ ' numbers of words are given as input; each word is taken one by one for categorized as either concept or relation and adding to the table. The time taken for processing this algorithm is ' $n$ ' unit time for sorting and appending.

The time complexity for Content Parsing Algorithm is $\mathrm{O}(\mathrm{n})$.

\section{B. Algorithm for Adding Nodes}

To take care of the issues in knowledge representation a powerful structure for symbolizing the data is required. A graphical structure is utilized to streamline this circumstance; consequently Concept Relation (CR) diagram is conveyed.

\section{Notation:}

Given a sentence table ST containing concepts $\mathrm{C}$ and relations $\mathrm{R}$ with their unique ids $\mathrm{c}_{-}$id and $\mathrm{r}_{-}$id respectively. Such that ST: $\{\mathrm{C} U \mathrm{R}\}$, where $\mathrm{CT}=\left\{\mathrm{C}_{1} \mathrm{UC}_{2} \mathrm{U} \ldots \mathrm{C}_{\mathrm{n}}\right\}$ and RT $=\left\{R_{1} U R_{2} U \ldots . . R_{m}\right\}$ are separated and placed in a unique position in the Concept Relation (CR) graph.

\section{Algorithm:}

Adding_Content(word1,..,wordn)

\section{Input: Content $\left(\operatorname{word}_{1}, \ldots\right.$, word $\left._{\mathrm{n}}\right)$ to be included from ST}

Output: Entire content added to the CR graph structure

/* Relation Table (RT) contains a set of relations */

/* Concept Table (CT) contains clauses, which are existing in the graph*/

\section{Procedure:}

DETERMINE the length of the content

INITIALIZE count $=0$

IF count $<$ length of the content

CHECK IF the word is in RT

RETURN (relation)

count++

ELSE

CHECK IF the word is present in CT

RETURN (concept)

count++

\section{ELSE}

\section{CHECK IF CT $\neq \phi$}

FIND the word position to be added

ADD the word to CT

count++

\section{END}

ADD the word to $\mathrm{CT}$ as position=1

count++

As a pre imperative, two tables are made. One table known as Relation Table (RT) is loaded with the relations. This RT is pre-characterized and changes can't be made. The other table is Concept Table (CT), which continues adding concepts to the table as new undefined words are added.[8]

At the point when the words captured are feed from the table, the quantity of words is calculated. Checking the count to be lesser than the quantity of words acquired for the table, the technique proceeds. Each and every word is taken independently and compared with the pre-characterized verb list in the RT. If the word present, then that word is marked as a relation and its position is returned. Otherwise, that word is verified with the clauses which are already added into the $\mathrm{CT}$. If the CT contains no word, then add this word as the first word. Moreover find out the most suitable location for adding the word in CT. Increase the count by one and repeat the same routine for the next word till all the words from the spreadsheet are placed in the correct position.

\begin{tabular}{|c|c|l|c|}
\hline s_id & c_id & concept & order \\
\hline 182 & 1488 & bad & 12 \\
\hline 182 & 1489 & habits & 13 \\
\hline 182 & 1470 & like & 14 \\
\hline 182 & 1471 & smoking & 15 \\
\hline 182 & 1472 & Drinking & 16 \\
\hline 182 & 1473 & drug & 18 \\
\hline 182 & 1474 & addiction & 19 \\
\hline 183 & 1475 & We & 1 \\
\hline 183 & 1476 & should & 2 \\
\hline 183 & 1477 & have & 3 \\
\hline 183 & 1478 & morning & 4 \\
\hline 183 & 1479 & walk & 5 \\
\hline 183 & 1480 & regularly & 6 \\
\hline 183 & 1481 & light & 7 \\
\hline 183 & 1482 & exercise & 8 \\
\hline 183 & 1483 & fresh & 10 \\
\hline 183 & 1484 & air & 11 \\
\hline 183 & 1485 & stroll & 14 \\
\hline 183 & 1486 & after & 15 \\
\hline 183 & 1487 & dinner & 16 \\
\hline
\end{tabular}

Fig. 5: Concept Table (CT) updated with Concepts

\begin{tabular}{|c|c|l|c|}
\hline s_id & $\mathbf{r}$ id & \multicolumn{1}{|c|}{ relation } & order \\
\hline 177 & 464 & and & 16 \\
\hline 178 & 465 & is & 2 \\
\hline 179 & 467 & in & 3 \\
\hline 179 & 468 & is & 5 \\
\hline 179 & 469 & a & 6 \\
\hline 180 & 470 & But & 1 \\
\hline 180 & 471 & to & 4 \\
\hline 180 & 472 & for & 7 \\
\hline 180 & 473 & a & 10 \\
\hline 181 & 474 & is & 3 \\
\hline 181 & 475 & on & 6 \\
\hline 181 & 476 & the & 7 \\
\hline 182 & 477 & For & 1 \\
\hline 182 & 478 & the & 11 \\
\hline 182 & 479 & and & 17 \\
\hline 183 & 480 & in & 9 \\
\hline 183 & 481 & and & 12 \\
\hline 183 & 482 & a & 13 \\
\hline 184 & 483 & be & 4 \\
\hline 184 & 484 & of & 6 \\
\hline
\end{tabular}

Fig. 6: Relation Table (RT) with Relations 


\section{Complexity:}

The parsed words are added to the respective tables if they are recently included (i.e.) Concept Table or Relation Table. The time taken for processing this algorithm is ' $n$ ' unit time for picking the words and adding the word to a particular table.

The time complexity for Adding Node Algorithm is $\mathrm{O}(\mathrm{n})$.

\section{Algorithm for Modifying Nodes}

The clauses in the graph can be changed or deleted. At the point such variation happens in appropriate substitution. At the point when a concept is modified, the relation existing between those nodes with alternate nodes should be carefully handled.

\section{Notation:}

In a Concept Table $\mathrm{CT}=\left\{\mathrm{C}_{1} \mathrm{UC}_{2} \mathrm{U} \ldots \mathrm{C}_{\mathrm{n}}\right\}$, given an element $\mathrm{C}_{\mathrm{i}}$ to be modified.

If $\mathrm{C}_{\mathrm{i}} \in \mathrm{CT}$, then $\mathrm{C}_{\mathrm{i}}=\mathrm{C}_{\mathrm{i}}{ }^{\prime}$, Where $\mathrm{C}_{\mathrm{i}}$ and $\mathrm{C}_{\mathrm{i}}{ }^{\prime}$ are old and new modified values of $\mathrm{C}_{\mathrm{i}}$.

\section{Algorithm:}

Modify_Concept(word,whatchange)

Input: Concept $(\mathrm{C})$ to be modified as $\left(\mathrm{C}_{\text {new }}\right)$

Output: Modified CR graph.

\section{Procedure:}

$$
\text { IF CT } \neq \phi
$$

IF Concept $(\mathrm{C}) \sqsubseteq \mathrm{CT}$

THEN C $=\mathrm{C}_{\text {new }}$ marked

ALL the relation $\left(r_{1}, \ldots, r_{n}\right)$ nodes associated with $C$ are

$$
\begin{aligned}
& \text { CHANGES are made as } \mathrm{C}_{\text {new }} \text { and }\left(\mathrm{r}_{1}, \ldots, \mathrm{r}_{\mathrm{n}}\right) \\
& \text { ELSE } \\
& \text { RETURN Concept }(\mathrm{C}) \text { not found in CT } \\
& \text { RETURN CT is Empty } \\
& \text { IF C } \subseteq \text { RT } \\
& \text { NO modification can be done } \\
& \text { ELSE } \\
& \text { RETURN Concept (C) not found in RT }
\end{aligned}
$$

If any change must be made to an element then its accessibility in the CT is checked. If it is available then all the relation nodes rising up out of this concept node are marked. Fundamental changes like replacement or deletion can be performed to those marked nodes.

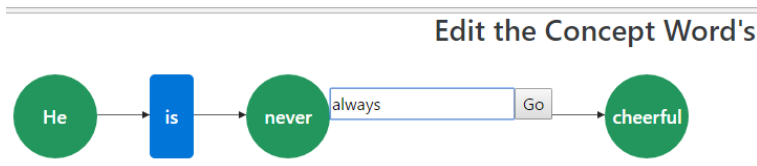

Fig. 7: Concept for Modification

If replacement of concept must be done, at that point the unique number must be changed. The newly replaced concept exist the concept list, then the corresponding unique number has to be updated in the concept table and sentence table, otherwise new unique number has to be generated.

For deletion of concept, the concept name along with unique number and sequence number has to be removed from the sentence table. If the concept is in the beginning of the sentence formed, then the entire sentence is deleted from the sentence table and necessary updates like change of unique number, concept number are made in the concept table.

If the word is found in RT, then no modification can be done because this table is not created by appending data but by pre defining the verbs as relations.

\section{Complexity:}

For checking the presence of a specific concept or relation in knowledge base, all the elements from both the tables are checked. Suppose ' $n / 2$ ' number of concepts is from Concept Table (CT) and ' $\mathrm{n} / 2$ ' number of relations is present in Relation Table (RT). Then the maximum number of comparison requested for identifying the word is ' $n / 2+n / 2=$ n'.

The time taken for figuring out the target node requires ' $n$ ' unit of time. Replacing the old word with a new word requires ' 1 ' unit of time (i.e.) rewriting the old with new concept.

The time complexity for Modifying Node Algorithm is $\mathrm{O}(\mathrm{n})$.

\section{Algorithm for Traversing Nodes}

Reasoning [9] assumes a more prominent part in the process of knowledge representation [10]. Therefore finding the occurrence of a concept and getting their relation with other concept fetches the model [11].

\section{Notation:}

In a Concept Table $\mathrm{CT}=\left\{\mathrm{C}_{1} \mathrm{UC}_{2} \mathrm{U} \ldots \mathrm{C}_{\mathrm{n}}\right\}$, given an element $\mathrm{C}_{\mathrm{i}}$ to be modified.

If $C_{i} € C T$, then $C_{i}$ is displayed along with other neighboring nodes.

\section{Algorithm:}

\section{Traverse_Content(word)}

Input: Concept $(\mathrm{C})$ to be searched

Output: Displays the related information.

\section{Procedure:}

IF CT $\neq \phi$

IF Concept $(\mathrm{C}) \sqsubseteq \mathrm{CT} \| \mathrm{RT}$

THEN the related Nodes are fetched

DISPLAY the concepts $\left(\mathrm{C}, \mathrm{C}_{1}, \mathrm{C}_{2}, . ., \mathrm{C}_{\mathrm{n}}\right) \& \&$ their relation $\left(r_{1}, \ldots, r_{n}\right)$

ELSE

RETURN Concept (C) not found RETURN CT is Empty

This algorithm sees if the clause is available in CT or RT. Once the presence is confirmed then the clause alongside the other related neighboring concepts and their relationship are taken out and displayed.[12] Or else the presence of the searched entity is denied.

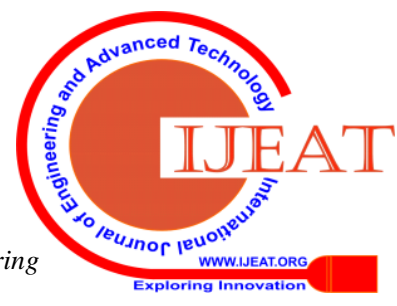




\section{A Knowledge Representation Model using Concept-Relation Graph}

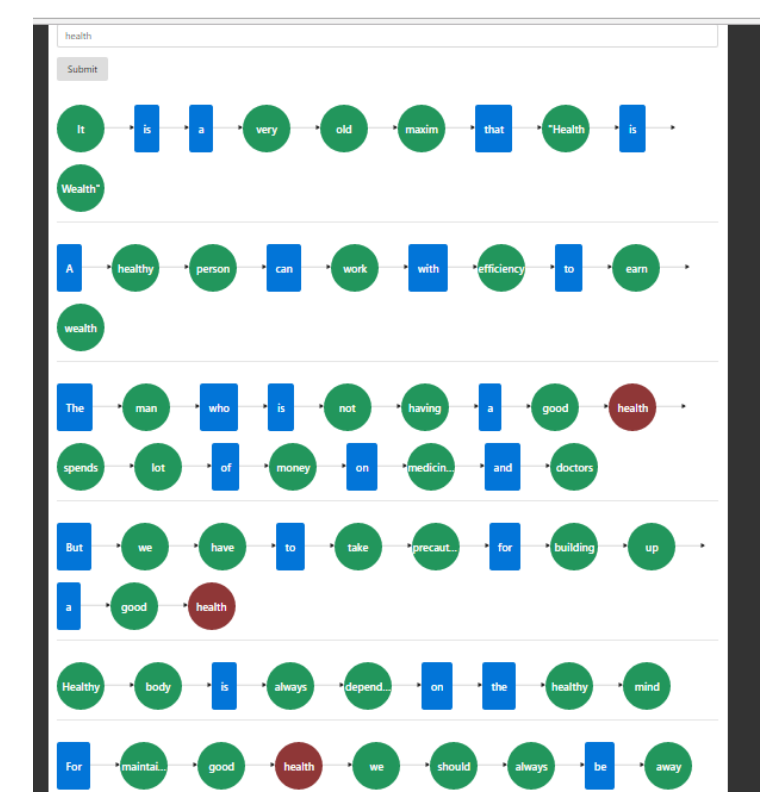

Fig. 8: Search for Concept

The extracted concepts if connected with more than one concept through a relation, at that point other concepts are also shown. Thus enabling the retrieval of every possible concept associated with the selected concept.

\section{Complexity:}

The time taken by this algorithm to determine the presence of a given word is checked in both Concept Table (CT) and Relation Table (RT), and to display if present is ' $n / 2+n / 2=$ n'.

The time complexity for Traversing Nodes Algorithm is $\mathrm{O}(\mathrm{n})$.

\section{CONCLUSION}

In this paper, we have presented the working of our knowledge representation model to demonstrate with different algorithms for content parsing the record, including, changing and searching concepts. The objective of this paper is to bring the content from an source which is semi organized, to place the content in a systematic way and to extract significant content from the knowledge base using Concept_Relation (CR) Graph.

Brief description of a knowledge representation model using description logic and conceptual graph is given along with the various algorithms for constructing the concept relation graph. The data is taken and processed, to yield adequate knowledge. The main advantages in our representation model are no prior knowledge about the scenario is required and no data training [13] is done. As the data is fed into the system, it is processed and the graph is constructed. This can be easily adapted to any environment for generating knowledge.

A few more enhancements like logical entailment and reasoning a situation can be done as future work. This can eventually give conceptual information.

\section{REFERENCES}

1. Frank van Harmelen, Vladimir Lifschitz and Bruce Porter,"Handbook of Knowledge Representation". 1st Edition: 2007; Volume 1.

2. Madalina Croitoru et al,"Graphical norms via conceptual graphs", Knowledge-Based Systems. 2012; 29: pp. 31-43.

3. John F Sowa, "Knowledge Representation: Logical, Philosophical and Computational Foundations". New York, PWS Publishing Co., 2000.

4. S. Praveena Rachel Kamala and Dr. S. Justus, "Towards MORK: Model for Representing Knowledge". I. J. Modern Education and Computer Science. 2016; pp.45-53.

5. Chuntao Jiang et al,"Text classification using graph mining-based feature extraction”. Knowledge-Based Systems. 2010; 23: pp. 302-308.

6. Praveena Rachel Kamala S and Justus S, "Concept - Relation Constructs for Knowledge Representation". International Journal of Control Theory and Applications. 2016; 9: pp. 463 - 473.

7. Tiago A. Almeida et al,"'Text normalization and semantic indexing to enhance Instant Messaging and SMS spam filtering”. Knowledge-Based Systems. 2016; 108: pp. 25-32.

8. Julia Hoxha et al, "Automated learning of domain taxonomies from text using background knowledge". Journal of Biomedical Informatics. 2016; 63: pp. 295-306.

9. Miao Fana et al,"Distributed representation learning for knowledge graphs with entity descriptions". Pattern Recognition Letters. 2016; 000: pp. 1-7.

10. Praveena Rachel Kamala S and Justus S, "Concept Relation Logic for Visualizing File Behavior in a Knowledge Representation Model". Inernational Journal of Engineering and Advanced Technology. 2019; 8: pp. $1732-1740$.

11. Misael Mongiovì et al,"Merging open knowledge extracted from text with MERGILO”. Knowledge-Based Systems. 2016; 108: pp. 155-167.

12. Antonio Jimeno Yepes et al, "Knowledge based word-concept model estimation and refinement for biomedical text mining". Journal of Biomedical Informatics. 2015; 53: pp. 300-307.

13. Alberto Tonon et al,"“Contextualized ranking of entity types based on knowledge graphs". Web Semantics: Science.Services and Agents on the World Wide Web. 2016; 37-38: pp. 170-183.

\section{Authors ProfiLe}

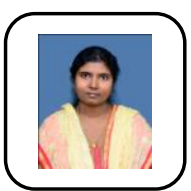

Praveena Rachel Kamala $\mathbf{S}$ is a full time $\mathrm{Ph} . \mathrm{D}$ Research Scholar at the School of Computer Science and Engineering, VIT University, Chennai, TN, India. Her areas of research include Knowledge Engineering, Knowledge Representation, Knowledge Storage and Retrieval.

She has received her undergraduate and postgraduate degrees in Computer Science \& Engineering from Madras University and Anna University, Chennai. She has worked as lecturer in several Engineering Educational Institutions in India.

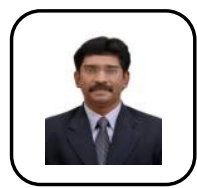

Dr. S. Justus received his doctorate degree from Madurai Kamaraj University, Madurai, India. His research specializations include Object-relational data modeling, knowledge engineering and Big Data

He has been into academic research and has published several of his research work results in International Journals and Conferences - including SwSTE in Israel and DASMA in Germany. He has also practitioner's experience while working with Software development companies.

$\mathrm{He}$ is a member of IEEE, ISTE, IAENG professional associations. He has served as research \& project coordinator for PG studies at Engineering Institutes. Presently he is working as Associate Professor at VIT University, Chennai. 